\title{
Pharmacopée traditionnelle de la région de Zerhoun - Maroc - : connaissances ancestrales et risques de toxicité
}

\author{
Mariame NAJEM*, Rajae BELAIDI, Ikram SLIMANI, El Houssine BOUIAMRINE, \\ Jamal IBIJBIJEN et Laila NASSIRI
}
Equipe de Microbiologie du Sol et de l'Environnement, Faculté des Sciences, Université Moulay Ismail, 50050, BP: 298, Marjane-Meknès-Maroc.
*Auteur correspondant; E-mail : mariamenajem@gmail.com ; B.P : 11201, Meknès, Maroc. Tel: (+212) 660530624

\section{RÉSUMÉ}

La population locale de la région de Zerhoun au Maroc a une riche tradition ancestrale dans le domaine de la phytothérapie. En effet, plusieurs plantes à vertus médicinales sont à l'origine d'intoxications constituant ainsi un problème de santé publique assez sérieux. Ainsi, le présent travail est une contribution à la connaissance des plantes toxiques ; il s'agit en effet de recenser parmi les plantes médicinales utilisées dans la région de Zerhoun, celles déclarées toxiques par la population locale. L'enquête a permis de déceler 17 plantes $(15,31 \%)$ sauvages sur la base de leurs noms vernaculaires locaux. Une prospection sur le terrain a été conduite dans un deuxième temps pour la collecte et l'identification préliminaire d'échantillons de ces plantes, Ensuite leur identification taxonomique a été faite en s'appuyant essentiellement sur la Flore marocaine. Les plantes à effet toxiques cataloguées appartiennent à 13 familles et utilisées essentiellement par voie interne, avec des doses aléatoires pour traiter une panoplie de maladies. Cette utilisation anarchique, non rationnelle des plantes médicinales présente un risque d'intoxication. La sensibilisation et la formation des utilisateurs et la législation dans le domaine de la phytothérapie traditionnelle s'imposent.

(C) 2018 International Formulae Group. All rights reserved.

Mots clés: Zerhoun, Pharmacopée traditionnelle, Plantes médicinales, Plantes toxiques.

\section{Traditional pharmacopoeia of the Zerhoun region - Morocco -: ancestral knowledge and risks of toxicity}

\begin{abstract}
The local population of the Zerhoun region of Morocco has a rich ancestral tradition in the field of herbal medicine. Indeed, several plants with medicinal properties are at the origin of intoxications thus constituting a rather serious public health problem. Thus, the present work is a contribution to the knowledge of toxic plants; it is a matter of identifying among the medicinal plants used in the region of Zerhoun, those declared toxic by the local population. The survey identified 17 wild plants $(15.31 \%)$ based on their local vernacular names. A field survey was conducted in a second time for the collection and preliminary identification of samples of these plants, then their taxonomic identification was made based mainly on the Moroccan Flora. The toxic effect plants cataloged belong to 13 families and used mainly internally, with random doses to treat a variety of diseases. This anarchic, non-rational use of medicinal plants presents a risk of intoxication. Awareness and user training and legislation in the field of traditional herbal medicine are needed. (C) 2018 International Formulae Group. All rights reserved.
\end{abstract}

Keywords: Zerhoun, Traditional pharmacopoeia, Medicinal plants, Toxic plants. 


\section{INTRODUCTION}

Depuis l'antiquité et à travers de nombreuses civilisations, la médication par les plantes a toujours été présente. En Afrique du nord, la phytothérapie est très ancrée dans la culture des populations et joue un rôle important en médecine traditionnelle (Bellakhdar, 2006).

Depuis les années 80, partout dans le monde, le recours aux plantes médicinales a connu un regain d'intérêt sans précédent. L'Organisation Mondiale de la Santé (OMS) estime que la médecine traditionnelle, notamment par les plantes, couvre les besoins en soins de santé de $80 \%$ de la population des pays en voie de développement (Bousta et Ennabili, 2011).

$\mathrm{Au}$ Maroc, pour les populations rurales, surtout celles éloignées des centres de santé, la demande en matière de traitement par les plantes est assez élevée ; aussi, cet usage des plantes reste en partie fondé sur l'idée que les plantes sont un moyen naturel de traitement dénué de tout risque. Or, une plante peut à la fois être utile et toxique.

La toxicité due à l'utilisation des plantes médicinales constitue un problème médical, et socio-économique ; c'est dans cette optique que nous avons exploré les résultats d'une enquête ethnobotanique réalisée auprès de la population locale de Zerhoun ; le but principal étant de contribuer à la connaissance de certaines plantes à effet toxique, parmi celles utilisées en médecine traditionnelle et de sensibiliser la population des risques d'intoxication et aux effets néfastes de toute utilisation non maîtrisée des plante.

\section{MATERIEL ET METHODES}

\section{Présentation de la zone d'étude}

La région de Zerhoun fait partie de la Wilaya de Meknès et couvre environ 55.800 ha ; elle est limitée au Nord par les provinces de Sidi Kassem et de Taounate, au Sud par la zone de Dkhissa, à l'Est par la province de Zouagha Moulay Yaakoub et à l'Ouest par la zone d'Ain Jemâa (DPAM, 2010). La population totale recensée en 2004 dans cette zone est de 54879 habitants est à dominance rurale (DPAM, 2010).

\section{Inventaire}

Durant une période étalée sur quatre mois, 19 Douars ont été investis, couvrant les six communes rurales de Zerhoun, (Walili, Kermet Ben Salem, N'zala Beni Amar, Cherkawa, Mghassyne, Sidi abdellah Lkhayat) et la commune urbaine, Moulay Idriss.

Moyennant la technique «Free listing » selon laquelle les répondants sont invités à énumérer les plantes médicinales locales qu'ils connaissent, un total de 350 fiches questionnaires (à raison d'une fiche par personne enquêtée) fut dûment rempli. Au cours d'entretiens individuels d'environ une heure chacun, des informations sur les plantes médicinales utilisées (identité vernaculaire, partie utilisée, mode de préparation, période de collecte, usages thérapeutiques et toxicité) ont été recueillies. Toutes les espèces citées ont été reportées par leurs noms communs.

\section{Analyses statistiques}

Par la suite, les données inscrites sur des fiches brutes ont été analysées par le logiciel de traitement statistique SPSS Statistics 20 et Excel 2010 pour faire ressortir toutes les informations relatives à la toxicité des plantes médicinales utilisées.

La liste des plantes déclarées toxiques, une fois établie, s'en est suivie une prospection sur le terrain, en collaboration avec le service des Eaux et Forêts, les plantes recensées lors l'enquête ethnobotanique furent repérées dans les différents sites de la région d'étude. $\mathrm{La}$ confirmation et/ou la détermination de leur taxonomie a été faite à la Faculté des Sciences de Meknès, sur la base de la Flore pratique du Maroc (Fennane et al., 1999; Fennane et al., 2007; Fennane et al., 2014). 


\section{RESULTATS}

Au terme de l'enquête ethnobotanique menée dans la région de Zerhoun, 111 plantes ont été recensées dont 17 ont été déclarées avoir un effet toxique, soit un pourcentage de $15,31 \%$. L'analyse floristique de la liste des 17 plantes rapportées toxiques a révélé leur répartition sur 13 familles, représentées chacune par une espèce, hormis, les Lamiaceae et les Liliaceae qui, elles, sont représentées respectivement par quatre et deux (Figure 1 et Tableau 1).

L'ensemble des plantes toxiques inventoriées, exceptée Salvia officinalis L., pousse spontanément dans la zone d'étude (Tableau 1 et Figure 2). Par ailleurs, les personnes enquêtées ont affirmé qu'elles ont recours à ces plantes pour guérir une panoplie de maladies : rhumatismales, gastriques, dermatologiques, métaboliques et dentaires (Tableau 1).

La dose utilisée pour chaque plante reste aléatoire : cuillerée $33,33 \%$, pincée $20,84 \%$ ou poignée $45,83 \%$, autant de doses imprécises (Tableau 1) ; à titre d'exemple, pour Aristolochia paucinervis Pomel., de son nom vernaculaire «Berztem », on peut trouver à la fois la poignée et la cuillerée. Pour sa part, l'enquête a révélé que la quasi-totalité des plantes signalées comme toxiques sont administrées par voie orale jusqu'à la guérison $73,68 \%$. Aussi, d'après les données présentées dans le tableau 1, la décoction est le mode de préparation le plus utilisé $(71 \%)$ suivi par l'usage de ces plantes sous leur état cru $(52,94$ $\%)$.

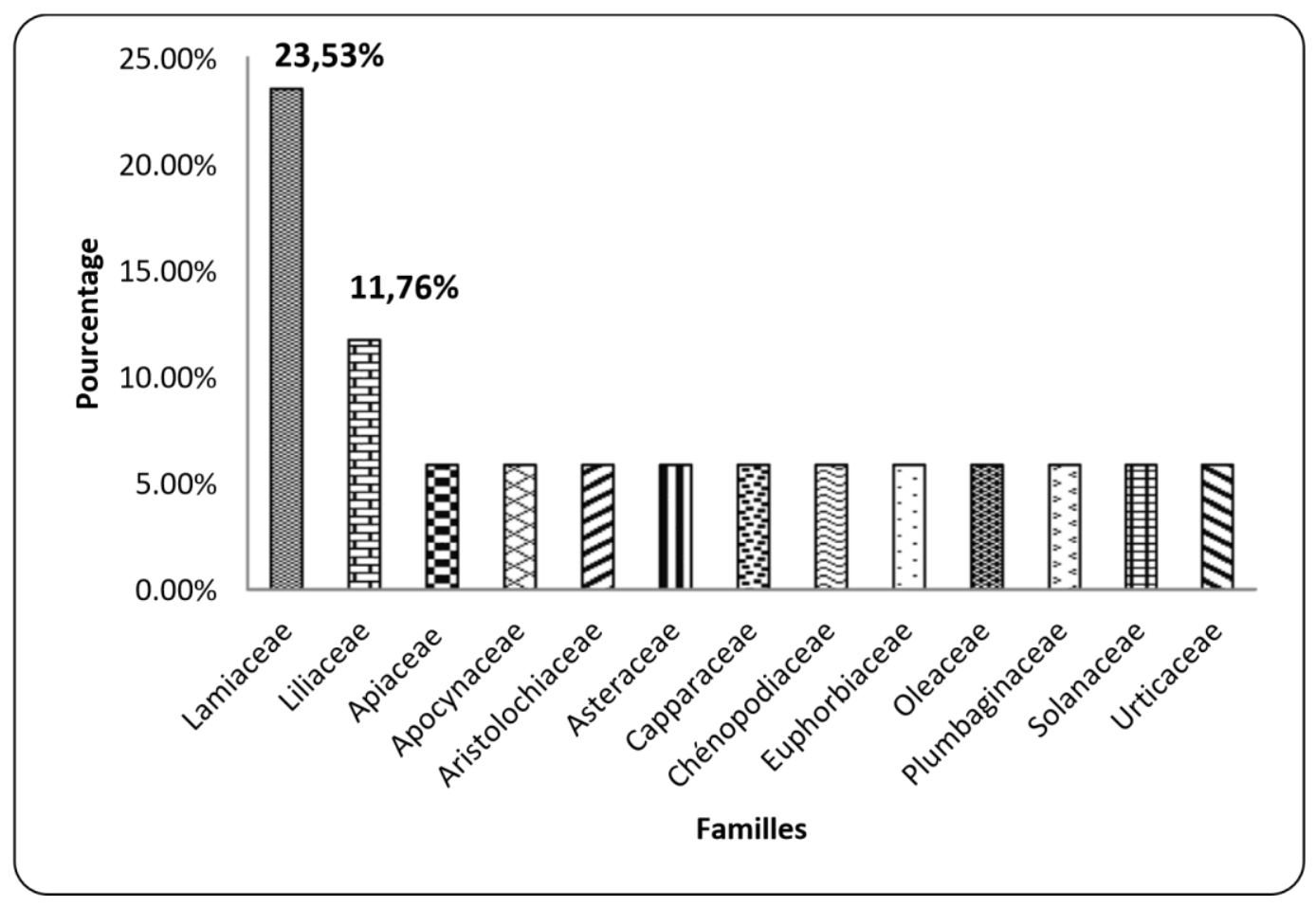

Figure 1: Répartition en pourcentage des familles desquelles relèvent les plantes médicinales déclarées toxiques. 
Tableau 1 : Inventaire des plantes médicinales déclarées avoir un effet toxique.

\begin{tabular}{|c|c|c|c|c|c|c|c|c|c|c|c|}
\hline Familles & $\begin{array}{c}\text { Noms } \\
\text { vernaculaires }\end{array}$ & Noms latins & Noms communs & T. pl & P.U & M.P. & M.A. & M.T. & Dose & D.T & $\begin{array}{c}\text { Pl } \\
\text { g }\end{array}$ \\
\hline $\begin{array}{l}\text { Apiaceae } \\
\text { (umbelliferae) }\end{array}$ & Bachnikha & Ammi visnaga (L.) Lam. & L'acanthe & Svg & $\mathrm{Fr}, \mathrm{Gr}$ & $\begin{array}{l}\text { Inf, } \\
\text { éc, Cru }\end{array}$ & Ora & Den & Pin & G & $\begin{array}{l}1 \\
f / j\end{array}$ \\
\hline Aristolochiaceae & Berztem & $\begin{array}{l}\text { Aristolochia paucinervis } \\
\text { Pomel=A.longa subsp } \\
\text { paucinervis (Pomel) batt }\end{array}$ & L'Aristoloche & Svg & $\mathrm{Ra}$ & Déc, Cru & Ora & $\begin{array}{l}\text { Mét, Der, } \\
\text { Gas, Rhu }\end{array}$ & Poi, Cuil & G & $\begin{array}{l}1 \\
f / j\end{array}$ \\
\hline Apocynaceae & Defla & Nerium oleander L. & Le laurier rose & Svg & $\mathrm{Fl}, \mathrm{Fe}$ & Déc, Cat & $\begin{array}{l}\text { Ora, Bad, } \\
\text { Fum }\end{array}$ & $\begin{array}{l}\text { Mét, Den, } \\
\text { Fvr }\end{array}$ & Pin & $\mathrm{S}$ & $\begin{array}{l}1 \\
\mathrm{f} / \mathrm{j}\end{array}$ \\
\hline $\begin{array}{l}\text { Asteraceae }= \\
\text { Compositae }\end{array}$ & Dad ; addâd & Atractylis gummifera L. & Le Chardon à glu & Svg & Pl.En & $\begin{array}{l}\text { Cat, Cru, } \\
\text { Cui }\end{array}$ & Bad, Fum & $\begin{array}{ll}\text { Der, } & \text { Rhu, } \\
\text { Abcès } & \end{array}$ & Poi, Cuil & G & $\begin{array}{l}1 \\
f / j\end{array}$ \\
\hline Capparaceae & $\begin{array}{l}\text { Kebbar; Taglulut; } \\
\text { Afsas }\end{array}$ & Capparis spinosa $\mathrm{L}$. & $\begin{array}{l}\text { Le Câprier } \\
\text { commun }\end{array}$ & Svg & $\mathrm{Fr}, \mathrm{Gr}$ & $\begin{array}{l}\text { Inf, } \\
\text { Cru }\end{array}$ & Ora, Rin & Ost, Rhu & Poi, Cuil & $\mathrm{G}$ & $\begin{array}{l}1 \\
f / j\end{array}$ \\
\hline Chénopodiaceae & Mkhinza & $\begin{array}{l}\text { Chenopodium } \\
\text { ambrosioides L. }\end{array}$ & L'Ambrosine & Svg & Fe, Pl.En & $\begin{array}{l}\text { Déc, Cat, } \\
\text { Cru }\end{array}$ & Ora, Bad & $\begin{array}{lr}\text { Den, } & \text { Der, } \\
\text { Gas, } & \text { Fvr, } \\
\text { maux de } & \text { tête }\end{array}$ & Poi & G & $\begin{array}{l}1 \\
f / j\end{array}$ \\
\hline Euphorbiaceae & Hriga melsa & Mercurialis anпиа L. & La Mercuriale & Svg & Pl.En & $\begin{array}{l}\text { Inf, } \\
\text { éc, Cat, } \\
\text { Cru }\end{array}$ & Ora, Bad & $\begin{array}{l}\text { Uro, } \\
\text { Mét,Der,Ga } \\
\text { s, Rhu }\end{array}$ & Poi, Cuil & $\mathrm{G}$ & $\begin{array}{l}3 \\
\mathrm{f} / \mathrm{j}\end{array}$ \\
\hline \multirow{2}{*}{ Lamiaceae } & Kohila & Lavandula multifida L. & $\begin{array}{l}\text { La lavande } \\
\text { multifide }\end{array}$ & Svg & Fe, Pl. En & Déc, Cui & Ora & $\begin{array}{l}\text { ORL, Gas, } \\
\text { Rhu, Res }\end{array}$ & Poi & $\mathrm{G}$ & $\begin{array}{l}2 \\
f / j\end{array}$ \\
\hline & Zaâtar; sahtar & $\begin{array}{l}\text { Origanum } \\
\text { compactum } \\
\text { Bentham. }\end{array}$ & $\begin{array}{l}\text { L'Origan } \\
\text { compact }\end{array}$ & Svg & $\begin{array}{l}\text { Fe, Pl. } \\
\text { En }\end{array}$ & $\begin{array}{l}\text { Inf, } \\
\text { Déc }\end{array}$ & Ora, Rin & $\begin{array}{l}\text { Uro, Der, } \\
\text { Gas, Res }\end{array}$ & Cuil,Pin & G & $\begin{array}{l}1 \\
f / j\end{array}$ \\
\hline
\end{tabular}


M. NAJEM et al. / Int. J. Biol. Chem. Sci. 12(6): 2797-2807, 2018

\begin{tabular}{|c|c|c|c|c|c|c|c|c|c|c|c|}
\hline & Halhal & Lavandula stoechas L. & $\begin{array}{l}\text { La lavande } \\
\text { stéchade; } \\
\text { Lavande papillon }\end{array}$ & Svg & $\begin{array}{ll}\mathrm{Fe}, & \mathrm{Pl} \\
\mathrm{En} & \end{array}$ & $\begin{array}{l}\text { Inf, Déc, } \\
\text { Cui, } \\
\text { Cat }\end{array}$ & Ora, Bad & $\begin{array}{ll}\text { Uro, } & \text { Der, } \\
\text { Gas, } & \text { Res, } \\
\text { Rhu } & \end{array}$ & Poi,Cuil & G & $\begin{array}{l}1 \\
\mathrm{f} / \mathrm{j}\end{array}$ \\
\hline & Salmia & Salvia officinalis L. & $\begin{array}{ll}\text { La } & \text { sauge } \\
\text { officinale } \\
\text { (cultivée) }\end{array}$ & $\begin{array}{l}\text { Svg, } \\
\text { clv }\end{array}$ & $\begin{array}{l}\text { Fe, } \\
\text { Pl. En }\end{array}$ & $\begin{array}{l}\text { Inf, } \\
\text { Déc }\end{array}$ & Ora, Rin & $\begin{array}{l}\text { Uro, Mét, } \\
\text { Gas, Rhu, S. } \\
\text { Chv, } \\
\text { calmant }\end{array}$ & Poi,Cuil & G & $\begin{array}{l}2 \\
\mathrm{f} / \mathrm{j}\end{array}$ \\
\hline \multirow{2}{*}{ Liliaceae } & Sekkoum; Tazzût & Asparagus officinalis L. & $\begin{array}{l}\text { L'Asperge } \\
\text { officinale }\end{array}$ & Svg & $\begin{array}{ll}\text { Ra, } & \mathrm{Fe}, \\
\text { Bou } & \end{array}$ & Déc, Cui & Ora & Gas, Rhu, & Poi & $\mathrm{G}$ & $\begin{array}{l}1 \\
\mathrm{f} / \mathrm{j}\end{array}$ \\
\hline & $\begin{array}{l}\text { Bassila; Ansal; } \\
\text { Bssel eddib }\end{array}$ & $\begin{array}{l}\text { Urginea maritima }(\mathrm{L} .) \\
\text { Baker }\end{array}$ & L’Urginée & svg & $\mathrm{Ra}$ & Cru, Cui & Ora, autre & ORL, Gas & Poi & $\mathrm{S}$ & $\begin{array}{l}1 \\
\mathrm{f} / \mathrm{j}\end{array}$ \\
\hline Oleaceae & $\begin{array}{l}\text { Azzaytoun el berri; } \\
\text { zebbouj }\end{array}$ & $\begin{array}{l}\text { Olea europea subsp } \\
\text { oleaster (Hoff \& Link) } \\
\text { Negodi }\end{array}$ & $\begin{array}{l}\text { L'Oléastre } \\
\text { L'Olivier } \\
\text { sauvage }\end{array}$ & Svg & $\mathrm{Fe}$ & Déc & $\begin{array}{l}\text { Ora, Rin, } \\
\text { Bad }\end{array}$ & $\begin{array}{l}\text { Mét, Den, } \\
\text { Gas, Rhu }\end{array}$ & Pin & $\mathrm{G}$ & $\begin{array}{l}1 \\
\mathrm{f} / \mathrm{j}\end{array}$ \\
\hline Plumbaginaceae & Swake raayan & Plumbago europea L. & $\begin{array}{l}\text { La } \\
\text { Dentellai } \\
\text { re; La Malherbe }\end{array}$ & Svg & $\begin{array}{l}\mathrm{Ra}, \mathrm{Fe}, \mathrm{Pl} . \\
\mathrm{En}\end{array}$ & Cat & Bad & $\begin{array}{l}\text { Ost, } \\
\text { Rhu }\end{array}$ & Pin & G & $\begin{array}{l}1 \\
\mathrm{f} / \mathrm{j}\end{array}$ \\
\hline Solanaceae & Bed l-ghul; Taryâla & $\begin{array}{l}\text { Mandragora autumnalis } \\
\text { Bertol= Mandragora } \\
\text { officinalis L. }\end{array}$ & La Mandragore & Svg & $\mathrm{Ra}, \mathrm{Fe}$ & Cat, Cru & Ora, Bad & $\begin{array}{l}\text { Ost, } \\
\text {, Rhu }\end{array}$ & Cuil & $\mathrm{J}, \mathrm{S}$ & $\begin{array}{l}1 \\
\mathrm{f} / \mathrm{j}\end{array}$ \\
\hline Urticaceae & Harrigua harcha & Urtica urens L. & L'Ortie brûlante & Svg & Fe, Pl.En & $\begin{array}{l}\text { Inf, } \\
\text { éc, Cat, } \\
\text { Cru }\end{array}$ & Ora, Bad & $\begin{array}{l}\text { Der,Gas, } \\
\text { Mét,Rhu, } \\
\text { S. Chv }\end{array}$ & Poi & S, G & $\begin{array}{l}2 \\
\mathrm{f} / \mathrm{j}\end{array}$ \\
\hline
\end{tabular}




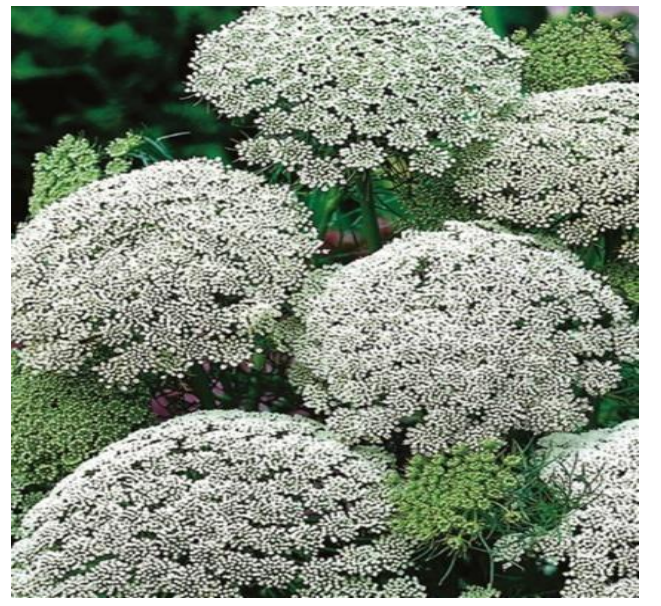

Aristolochia paucinervis Pomel=A.longa subsp

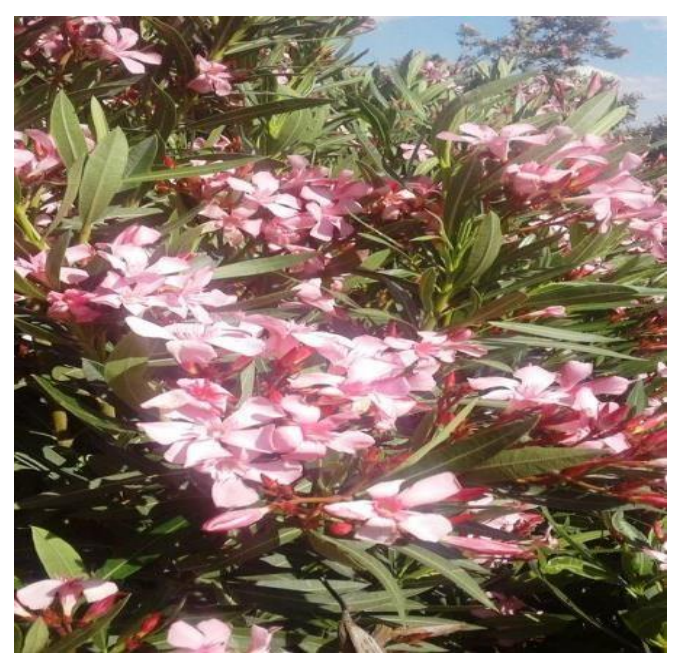

Nerium oleander L.

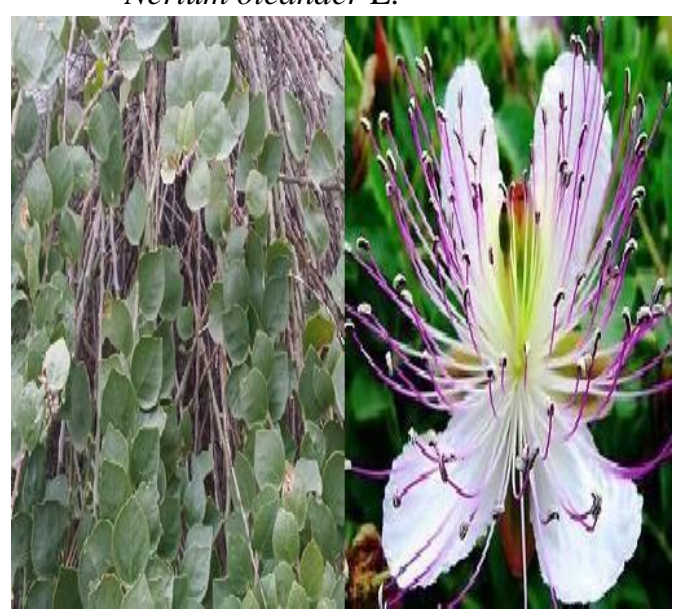

Chenopodium ambrosioides $\mathrm{L}$.

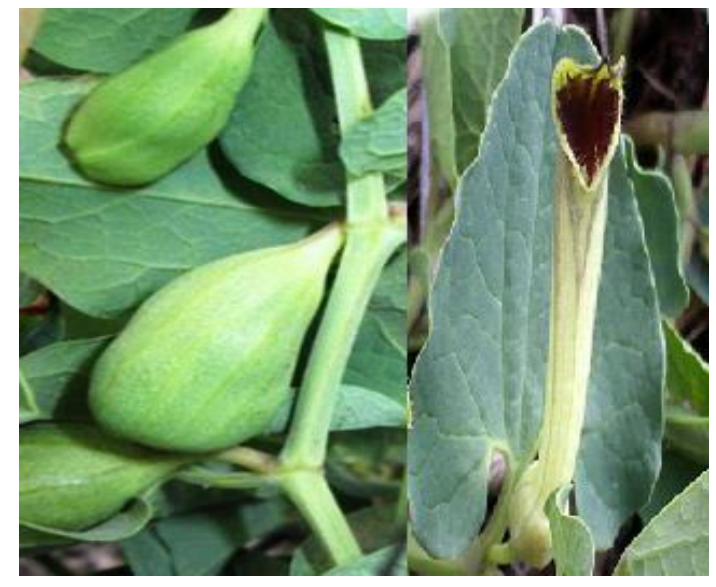

Ammi visnaga (L.) Lam. paucinervis (Pomel) batt

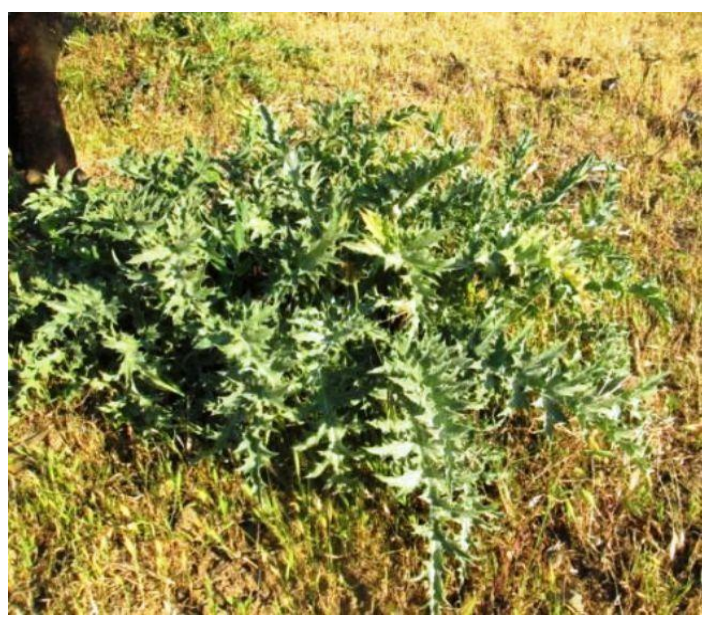

Atractylis gummifera $\mathrm{L}$

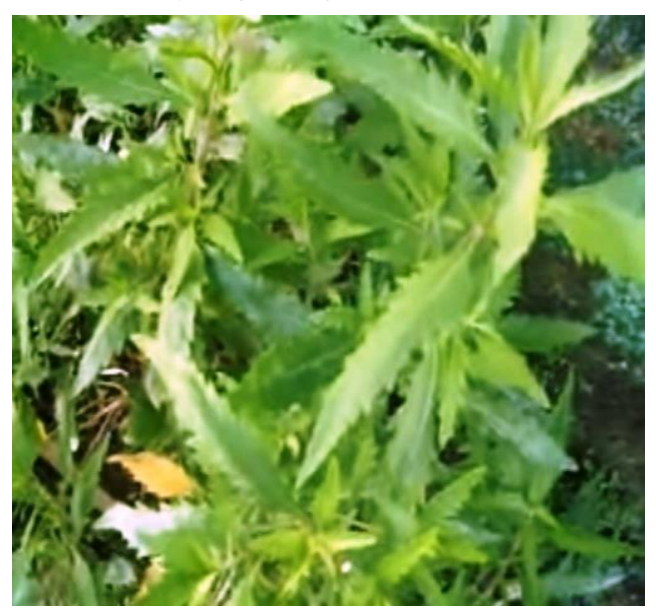

Chenopodium ambrosioides L. 


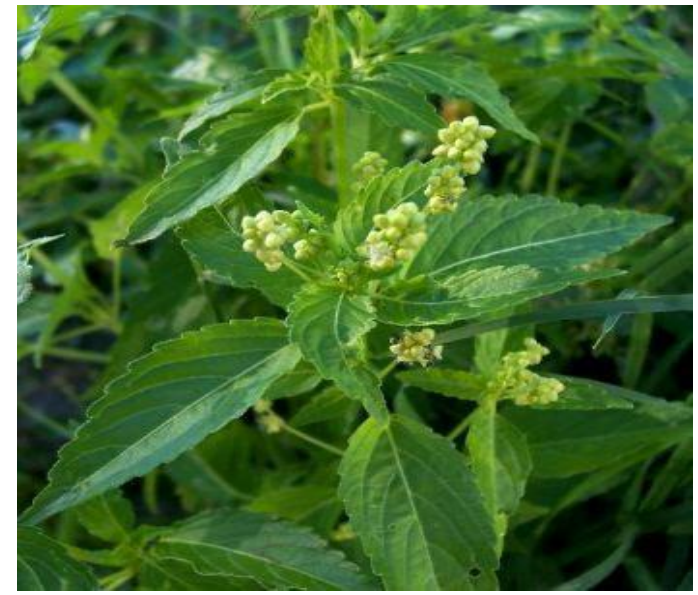

Mercurialis annua L.

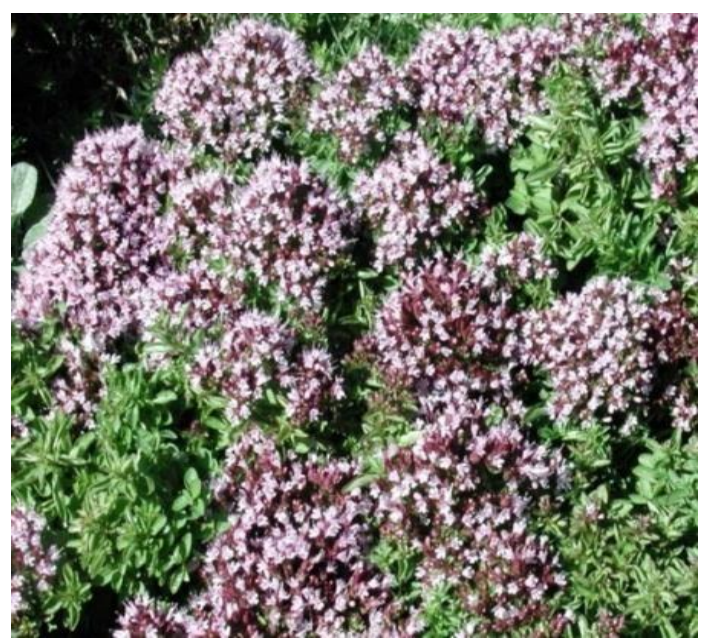

Origanum compactum Bentham.

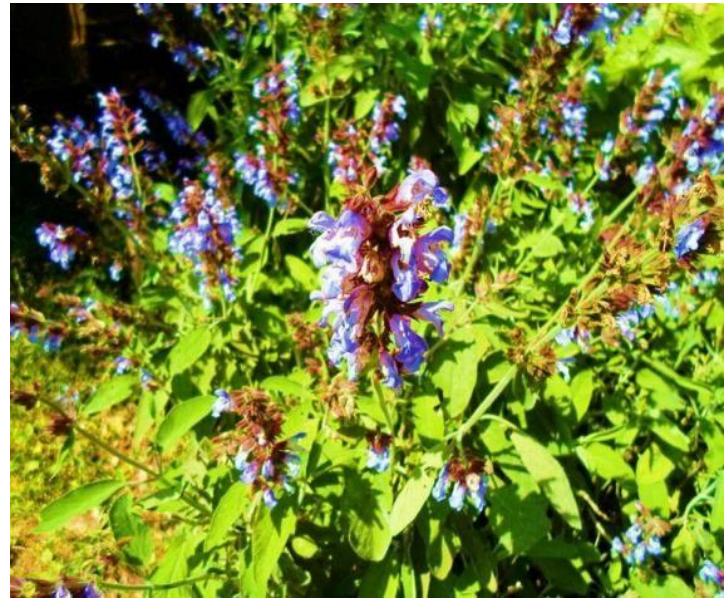

Salvia officinalis L.

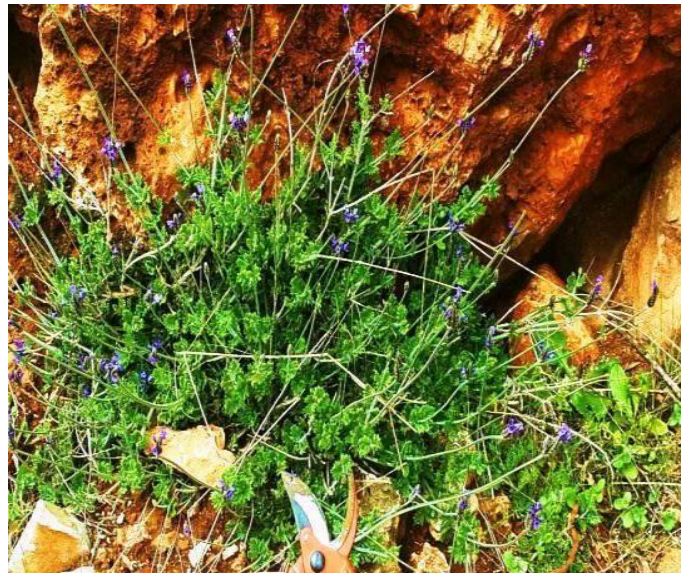

Lavandula multifida L.

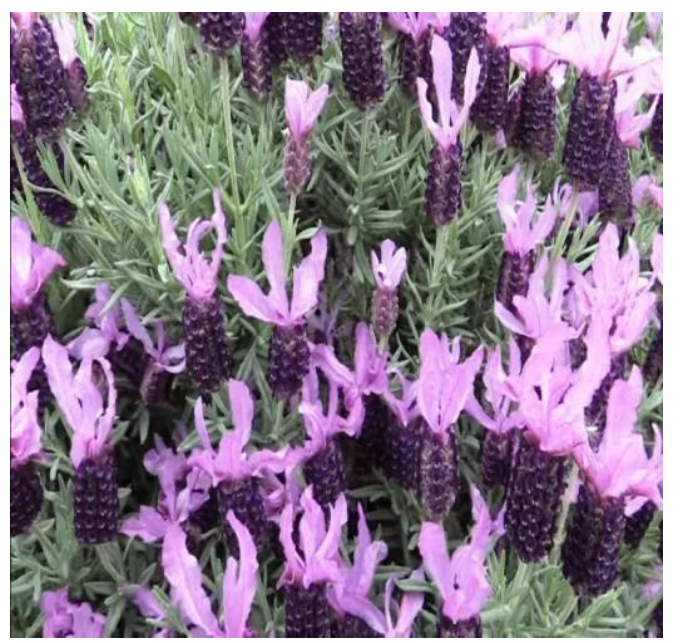

Lavandula stoechas L.

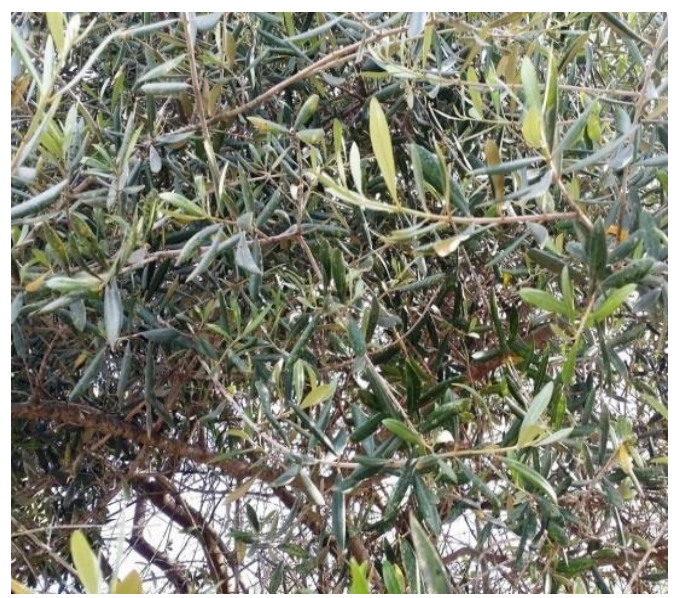

Olea europea subsp oleaster (Hoff \& \& Link) Negodi 


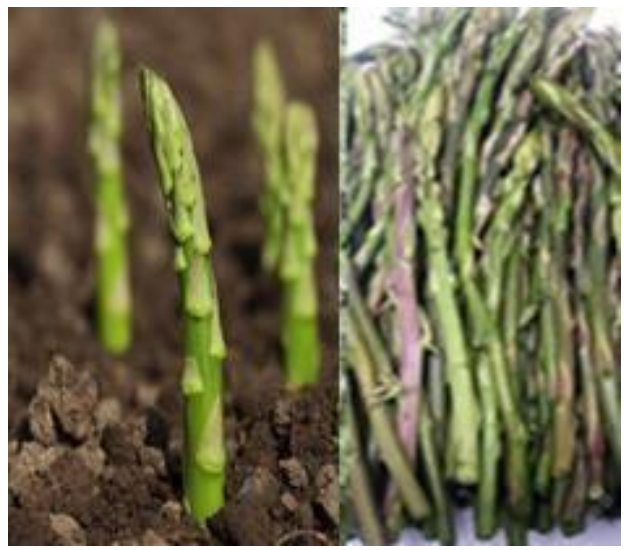

Asparagus officinalis L.

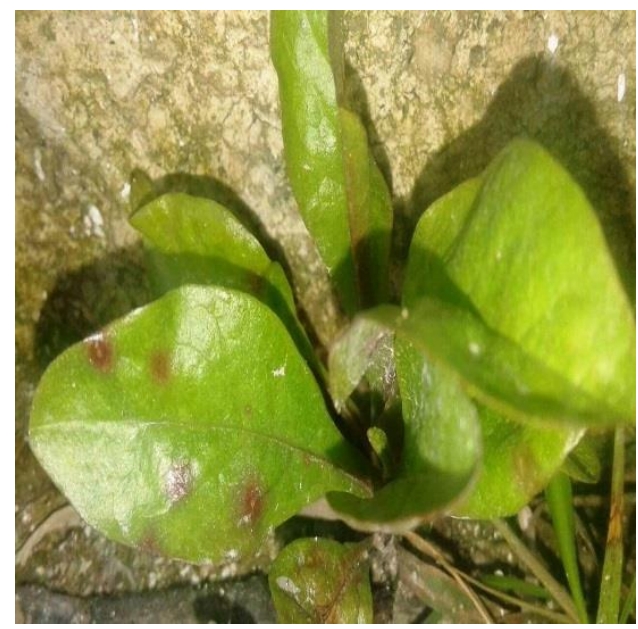

Plumbago europea L.

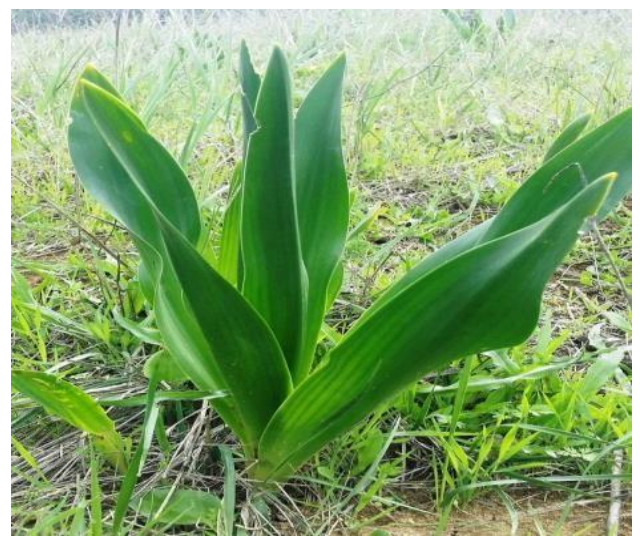

Urginea maritima (L.) Baker

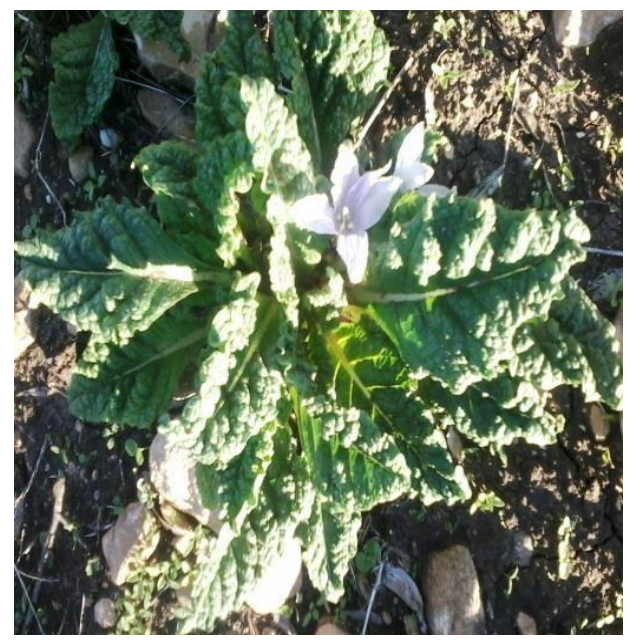

Mandragora autumnalis Bertol = Mandragora officinalis L.

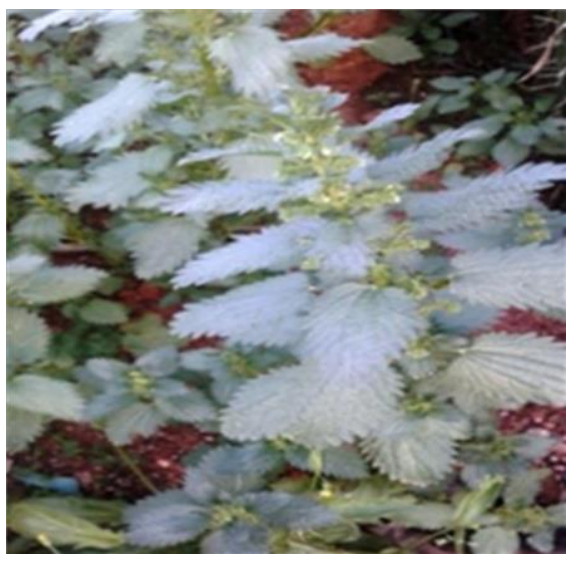

Urtica urens L.

Figure 2: Photos des plantes médicinales toxiques inventoriées au Zerhoun. 


\section{DISCUSSION}

La région de Zerhoun abrite un site d'intérêt biologique et écologique "SIBE de Zerhoun », réputé par sa richesse floristique, notamment en plantes médicinales (Slimani et al., 2016). Ainsi, la population locale a des connaissances ancestrales au terme de médication par les végétaux. Toutefois, et en dépit des vertus thérapeutiques des plantes médicinales, les enquêtés ont affirmé la toxicité de certaines espèces. Dans une étude pareille menée dans la région du Moyen Atlas central, une vingtaine de plantes toxiques a été révélée (soit un pourcentage de 19,82\%) parmi celles recommandées par les acteurs de la médecine traditionnelle à la population locale (Najem et al., 2018). De ce fait, la médecine douce doit être pratiquée avec précaution et à l'intérieur des paramètres et des mesures bien précises (Benlamdini et al., 2014).

Cependant, l'enquête a dévoilé l'utilisation anarchique de certaines espèces, accentuant encore plus leurs effets indésirables ; ainsi l'absence de notion de dose exacte chez la population locale peut se manifester par des effets néfastes sur la santé ; la toxicité dose-dépendante étant omniprésente (Benkhnigue et al., 2011). En effet, l'usager peut s'intoxiquer imprudemment, suite à un surdosage thérapeutique. Un végétal qui, à faible dose et dans des conditions rigoureuses d'emploi est médicinal, peut devenir une menace pour la santé de l'Homme s'il n'est pas utilisé correctement (Hammiche et al., 2013). Ainsi, Salvia officinalis L., est une plantes médicinale efficace à faible dose mais très toxique à forte dose (CAPM, 2016).

Par ailleurs, la meilleure utilisation d'une plante serait celle qui en préserverait ses propriétés tout en permettant l'extraction et l'assimilation des principes actifs (Dextreit, 1984). En général, l'administration des remèdes se fait soit par voie interne (infusion, décoction, extrait, hydrolat, etc...) ou externe (cataplasme, liniment, emplâtre, etc...) et certaines plantes peuvent même avoir, à la fois, un usage interne et externe (El Chaar et Apostolides, 2005). Or, l'utilisation fréquente du décocté peut être expliquée par le fait que la décoction permet de recueillir le plus de principes actifs (Slimani et al., 2016). Des études poussées dans ce sens doivent être faites pour vérifier si la décoction atténue ou annule l'effet toxique de certaines recettes comme cela a été souvent rapporté (Slimani et al., 2016). Le cas échéant, le risque est alors grand pour les plantes consommées crues. Aussi, les séquelles de la toxicité sont sûrement plus graves lors d'usage interne, l'intoxication étant généralisée. Donc, la durée d'utilisation, le mode de préparation et le mode d'administration sont autant de paramètres clés, qui s'ils ne sont pas maitrisés et respectés, peuvent générer des effets nocifs même pour une plante médicinale par excellence (Najem et al., 2018).

En fait, l'utilisation des plantes médicinales essentiellement sur la base de connaissances oralement transmises par les ancêtres, qui même s'ils ont au fil du temps découvert les vertus des plantes, ne maîtrisent pas les données relatives à leur phytochimie ni leurs propriétés «pharmaco-toxicologiques » aceroît leurs risques pour les usagers (Hammiche et al., 2013). Par conséquent, la nature du principe actif est un facteur évident qui influence l'activité pharmacologique ou toxicologique d'une plante. Les principes actifs d'origine végétale sont extrêmement variés, tant du point de vue structures moléculaires qu'impacts biologiques, ce qui confère à la plante son activité thérapeutique ou toxique. les plus dangereux sont surtout les alcaloïdes, les hétérosides cardiotoniques, les terpénoïdes des huiles essentielles; en second lieu arrivent les quinones, les saponosides, les furanocoumarines, les glucosinolates, les polyines et les oxalates de calcium (Alison et Paul, 2008) qui ont des actions diverses sur les différents système du corps. De ce fait, des études phytochimiques poussées des plantes recensées s'imposent pour mieux identifier les principes responsables des effets nuisibles.

Les effets indésirables et les signes d'intoxication diffèrent d'une plante à une autre. À titre d'exemple, Mandragora autumnalis Bertol et à cause de sa teneur en divers alcaloïdes dont les principaux sont la mandragorine, l'hyosciamine et l'atropine provoque une irritation gastro-intestinale, des hallucinations et un syndrome anticholinergique. La mort peut survenir en 12 
h, précédée ou non de coma (Piccilo et al., 2002). Plusieurs accidents et même des décès ont été signalés au Maroc, dans les compagnes, surtout chez les enfants, attirés par l'aspect luisant et la belle couleur jaune orange des baies (Bellakhdar, 1997).

Aussi, l'empoisonnement par Aristolochia paucinervis Pomel. est manifesté par l'altération des enzymes hépatiques et rénales, une hématurie et une paralysie des membres (Bnouham et al., 2006).16\% des cas d'insuffisance rénale ont été enregistrés chez les patients atteints de tumeurs malignes qui ont utilisé ladite plante pendant le traitement du cancer, et ce, selon les statistiques réalisées en 2010 par le Centre Anti-Poison du Maroc (Benzakour et al., 2011).

La symptomatologie de l'intoxication par Nerium oleander L. comporte : des céphalées, des frissons, l'irritation des muqueuses buccales, d'hypersalivation, des nausées, de gastroentérite, bradycardie, troubles de la conduction, arythmie, de risque de convulsions et parfois de mydriase importante, des risques de collapsus et de mort brutale. En cas d'application cutanée elle provoque des brûlures chimiques (Bakkali et al., 2010).

Face aux intoxications incontournables des plantes, les usagers devraient tenir certaines conduites. La plus importante est l'identification du végétal incriminé, la deuxième mesure est celle de l'appréciation de la quantité susceptible d'avoir été ingérée. Seul un interrogatoire précis des circonstances permet de faire une évaluation approximative de la quantité. Enfin, la connaissance du délai entre l'ingestion supposée et la consultation médicale est également un élément important à prendre en compte (Flesch, 2012). En milieu hospitalier, l'identification de la plante doit permettre, avec la symptomatologie, la prise de décision et la conduite à tenir (Hammiche et al., 2013). L'évacuation digestive et l'administration de charbon activé peuvent être préconisées en cas d'ingestion d'une quantité importante d'une plante toxique ou très toxique (Flesch, 2012).

\section{Conclusion}

Dans le cadre de la contribution à la connaissance des plantes médicinales à effet toxique, l'enquête menée auprès de la population locale de Zerhoun, a mis en évidence 17 plantes déclarée toxique utilisées en pharmacopée traditionnelle de la région.

L'ampleur du phénomène est sous-évaluée autant que ces plantes sont utilisées anarchiquement. Aussi, la grande diversité de ces plantes rend la symptomatologie observée extrêmement polymorphe suivant le toxique en cause. Les meilleures mesures resteront donc et sans équivoque la prévention et la sensibilisation. Pour cela, la communauté scientifique et les professionnels de santé, ensemble, doivent œuvrer pour la sensibilisation des citoyens quant aux risques potentiels de l'utilisation irrationnelle des plantes médicinales.

Les résultats de la présente étude pourraient constituer l'ébauche d'une base de données pour des recherches ultérieures visant à exploiter ces plantes toxiques, autrement qu'en médication, notamment comme alternatives biologiques aux pesticides.

\section{CONFLIT D'INTERETS}

Les auteurs déclarent qu'il n'y a aucun conflit d'intérêts.

\section{CONTRIBUTIONS DES AUTEURS}

$\mathrm{NM}, \mathrm{BR}$ et SI ont réalisé l'enquête, BEL a assuré les sorties de terrain et la prise des photos; IJ a assuré la typologie et a participé à enrichir le manuscrit et NL a supervisé le travail et a assuré l'identification botanique et taxonomique des plantes. Tous les auteurs ont participé à la rédaction du document.

\section{REMERCIEMENTS}

Au terme de ce travail, nous tenons à remercier les enquêtés de la zone d'étude pour le temps, la disponibilité et toutes les informations fournies.

\section{REFERENCES}

Alison J, Paul D. 2008. Toxicologie d'Urgence ( $1^{\text {ère }}$ edn). Elsevier/Masson : Paris.

Bakkali H, Ababou K, Nassim Sabah T, Moussaoui A, Ennouhi A, Fouadi F.Z, Siah S, Ihrai H. 2010. Les brulures chimiques par le laurier rose. Annals of Burns and Fire Disasters, 23(3) : 128130. 
Bellakhdar J. 1997. La Pharmacopée Marocaine Traditionnelle, Médecine Arabe Ancienne et Savoirs Populaires. Éditions Le Fennec : Casablanca.

Bellakhdar J. 2006. Plantes Médicinales au Maghreb et Soins de Base, Précis de Phytothérapie Moderne. Éditions Le Fennec : Casablanca.

Benkhnigue O, Zidane L, Fadli M, Elyacoubi H, Rochdi A, Douira A. 2011. Étude ethnobotanique des plantes médicinales dans la région de Mechraâ Bel Ksiri (Région du Gharb du Maroc). Acta Botanica Barcinonensia, 53: 191-216.

Benlamdini N, Elhafian M, Rochdi A, Zidane L. 2014. Étude floristique et ethnobotanique de la flore médicinale du Haut Atlas oriental (Haute Moulouya), Maroc. Journal of Applied Biosciences, 78: 6771-6787. DOI: http://dx.doi.org/10.4314/jab.v78i1.17

Benzakour G, Benkirane N, Amrani M, Oudghiri M. 2011. Immunostimulatory potential of Aristolochia longa L. induced toxicity on liver, intestine and kidney in mice. Journal of Toxicology and Environmental Health Sciences, 3(8): 214-222. DOI: 10.5897/JTEHS

Bnouham M, Merhfour FZ, Elachoui M, Legssyer A, Mekhfi H, Lamnaouer D, Ziyyat A. 2006. Toxic effects of some medicinal plants used in Moroccan traditional medicine. Moroccan J. Biol., 2(3) : 21-30.

Bousta D, Ennabili A. 2011. L'Institut national des plantes médicinales et aromatiques au service du développement de la phytothérapie au Maroc. Phytothérapie, 9(5): 297-303. DOI: 10.1007/s10298-0110658-x

CAPM. 2016. Centre Antipoison et de Pharmacovigilance du Maroc. Ministère de la santé. Royaume du Maroc.

Dextreit R. 1984. La Cure Végétale, toutes les Plantes pour se Guérir ( $3^{\text {ème }}$ edn). Éditions de la Revue Vivre en Harmonie : Paris.

DPAM . 2010. Étude monographique de la région Zerhoun. Direction Provinciale de l'Agriculture de Meknès.

El Chaar S, Apostolides NA. 2005. Étude ethnobotanique et éthopharmacologique des espèces médicinales libanaises agissant sur le système bronchopulmonaire. Annales de Recherche Scientifique, 6: 145-176.

Fennane M, Ibn Tattou M, El Oulaidi J. 2014. Flore pratique du Maroc. Vol. 3, Travaux Institut Scientifique. Sér. Botanique $\mathrm{N}^{\circ}$ 40 : Rabat.

Fennane M, Ibn Tattou M, Mathez J, Ouyahya A, El Oulaidi J. 1999. Flore pratique du Maroc. Vol. 1, Travaux Institut Scientifique. Sér. Botanique $\mathrm{N}^{\circ} 36$ : Rabat.

Fennane M, Ibn Tattou M, Ouyahya A, El Oulaidi J. 2007. Flore pratique du Maroc (Vol. 2), Travaux Institut Scientifique. Sér. Botanique, $\mathrm{N}^{\circ} 38$ : Rabat.

Flesch F. 2002. Plantes toxiques : les dangers du retour à la nature. Réanimation, 21 (5): 525-532. DOI: $10.1007 / \mathrm{s} 13546-012-$ 0494-5

Hammiche V, Merad R, Azzouz M. 2013. Plantes Toxiques à Usage Médicinal du Pourtour Méditerranéen. SpringerVerlag: Paris, France.

Najem M, Belaidi R, Harouak H, Bouiamrine EH, Ibijbijen J, Nassiri L. 2018. Occurrence de plantes toxiques en phytothérapie traditionnelle dans la région du Moyen Atlas central Maroc. Journal of Animal \& Plant Sciences, 35(2): 5651-5673.

Piccilo G, Mondati E, Moro P. 2002. Sixclinical cases of Mandrogora autumnalis poisoning: diagnosis and treatment. Eur. J. Emerg. Med. 9(4):3427.

DOI: 10.1097/01.mej.0000043855.56375.a7

Slimani I, Najem M, Belaidi R, Bachiri L, Bouiamrine EH, Nassiri L, Ibijbijen J. 2016. Étude ethnobotanique des plantes médicinales utilisées dans la région de Zerhoun -Maroc-. International Journal of Innovation and Applied Studies, 15(4): 846-863. 